\title{
Wide Band Ka-Band Coupled-Cavity Traveling Wave Tube (CCTWT) Development
}

\author{
Michael Cusick, Rasheda Begum, Deepika Gajaria, Tom Grant, Peter Kolda, Jim Legarra, \\ Brad Stockwell
}

Microwave Power Products Division

Communication and Power Industries LLC

Palo Alto, CA 94304 [email: michael.cusick@cpii.com]

David K. Abe, Igor A. Chernyavskiy, Mike Daniell, Baruch Levush, John Pasour,
Alexander. N. Vlasov
Naval Research Laboratory
Washington, DC 20375 [email: david.abe@nrl.navy.mil]

Alexander T. Burke, David P. Chernin, John J. Petillo

SAIC

McLean, VA 22102 and Billerica, MA 01821

\begin{abstract}
A series of Ka-band Coupled-Cavity TravelingWave Tubes (CCTWTS) has been successfully developed, built, and tested at Communications and Power Industries (CPI) in collaboration with the Naval Research Laboratory (NRL) and SAIC. These devices represent a significant advance in the state-of-the-art of millimeter-wave CCTWTs, exploring the limits of power, bandwidth, and stability. We discuss the design and successful demonstration of the series of CCTWT's, including the VTA-6430N1 prototype which achieved over 700-W (879-W maximum power) over a 5-GHz range in Ka-band.
\end{abstract}

Keywords: coupled-cavity traveling-wave tube; CCTWT, high power amplifier; broadband; periodic permanent magnet (PPM); large-signal TWT codes.

\section{Introduction}

There is a critical need for compact, broadband, high power millimeter-wave amplifier technology that supports a wide array of applications including high data rate communications, radar, and electronic warfare. The CCTWT is an attractive device type for these applications since it supports high peak and average power with good efficiency and moderate bandwidths.

This work describes recent advances in PPM focused coupled-cavity TWT design that have significantly increased the power-bandwidth performance of Ka-band devices. These devices have the potential to be the enabling technology for new millimeter-wave transmitters.

At the start of this development effort, the current state-ofthe-art in periodic permanent magnet (PPM) focused CCTWTs operating in Ka-band at CPI was $\sim 1-\mathrm{kW}$ peak power at $30 \%$ duty with a bandwidth of $\sim 1-\mathrm{GHz}$ for pulsed devices and $\sim 500$-W power with a bandwidth of $\sim 2-\mathrm{GHz}$ for $\mathrm{CW}$ devices. Utilizing the recent advances of NRL based 1-D and 2-D multi-frequency codes and commercial 3-D electromagnetic codes, we have created hardware prototypes to demonstrate design improvements and to provide experimental validation of the codes.

\section{Simulation Codes}

To better understand and design the physical mechanisms that affect the performance of these CCTWTs, we relied on CPI's in-house 1-D code and NRL based 1-D and 2-D multi-frequency large-signal codes CHRISTINE-CC [1] and TESLA-CC [2], the 3-D beam optics code MICHELLE [3], the commercial 3-D electromagnetics codes HFSS (Ansoft Corp.) and Analyst (STAAR/AWR). These codes enable a better understanding of the circuit performance prior to completing the design and procuring piece parts.

\section{Broadband CCTWT Prototypes}

The starting point for broadband development was the CPI VTA-6430A1 CCTWT which has a nominal output power of $500-\mathrm{W} \mathrm{CW}$ and an instantaneous bandwidth of $1-\mathrm{GHz}$ (30 to $31 \mathrm{GHz}$ ). The broadband prototypes developed in this effort used the same electron gun, PPM focusing system, and general form factor as the VTA-6430A1 but a succession of component and circuit modifications was made to increase the power and bandwidth. In all cases, the input and output coupler, sever, and circuit matches were redesigned to accommodate the increased operational bandwidth. In total, six broadband CCTWT's were built, NRL-1, NRL-2, NRL-3, VTA-6430N1, VTA-6430N1A and VTA-6430N2.

NRL-1, -2 and -3 were designed with a $30.0 \mathrm{GHz}$ center frequency and $5 \mathrm{GHz}$ operational bandwidth. The goal of these three CCTWT's was to successively improve power $\times$ bandwidth performance. VTA-6430N1, 6430N1A and $6430 \mathrm{~N} 2$ utilized the experienced gained with the NRL series CCTWTs. The goal of these three VTA-series CCTWTs was to operate across $5 \mathrm{GHz}$, at a higher center frequency than the NRL series CCTWTs, and to meet or exceed $700 \mathrm{~W} \mathrm{CW}$ output power. 


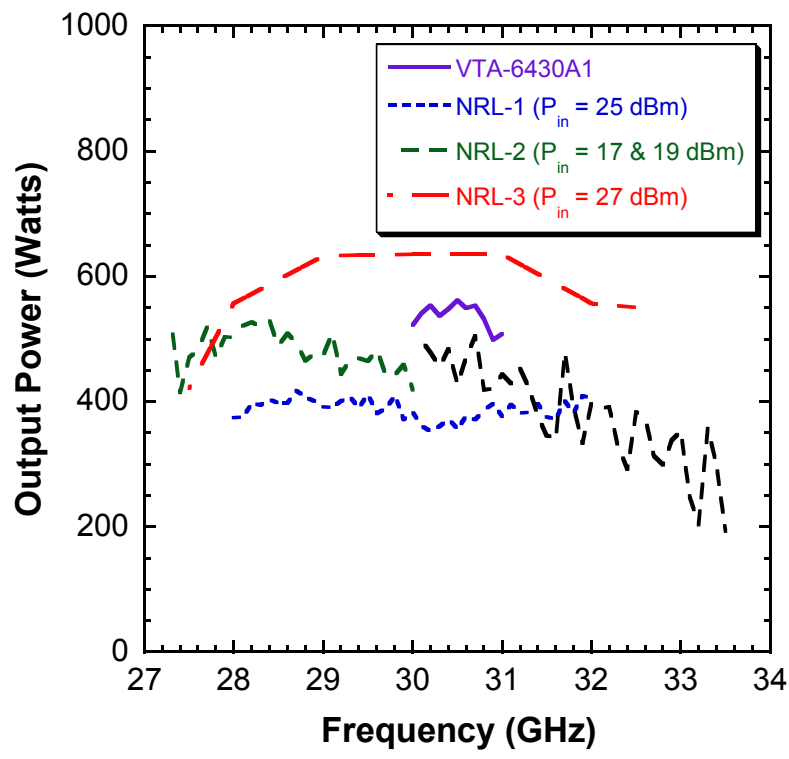

Figure 1: Output power vs. frequency (constant input drive) for VTA-6430A1 and NRL-1, -2, and -3 .

\section{NRL-1, -2 and -3 Prototypes}

Figure 1 plots the output power (constant input drive) versus frequency for the NRL-1, -2 and-3 prototypes; for reference, the performance of the initial commercial CCTWT, VTA-6430A1 (solid line), is also included. (NRL-2 was taken at two input drive powers, resulting in the split plot). Compared with VTA-6430A1, the modifications to the coupling slots resulted in a more than five-fold increase in bandwidth ( $>5 \mathrm{GHz}$ ). NRL-1 produced a maximum power of $480-\mathrm{W} C W$ without Drive Induced Oscillation (DIO) $\left(P_{i n}=29 \mathrm{dBm}\right)$. NRL-2 achieved a maximum of $540-\mathrm{W}$ CW before DIO was observed. NRL-3 exhibited a DC instability (subsequent analysis revealed that the beam coupled to the circuit mode at the lower end of the pass band, $\pi$ phase shift point), however, it was possible to drive through this instability with the application of RF to the circuit and a maximum output power of $635-\mathrm{W}$ was achieved.

The NRL-1, -2 and -3 prototypes explored the limits of output power achievable with the $16.48 \mathrm{kV}, 420 \mathrm{~mA}$ electron beam; the onset of DIO placed an upper limit of $635-\mathrm{W}$ from these devices.

\section{VTA-6430N1, 6430N1A and 6430N2 Prototypes}

The VTA-6430N1, 6430N1A and 6430N2 prototypes were a direct follow-on to the NRL-1, -2 , and -3 prototypes. To further improve the DIO margin, the cathode voltage and current were increased to $\sim 19.3 \mathrm{kV}$ and $500 \mathrm{~mA}$.

Figure 2 plots the output power (constant input drive solid line) (varied drive - dashed line) versus frequency for the VTA-6430N1 and 6430N2 CCTWTs. As seen in the plot, the modifications to the velocity step and increase in the beam voltage and current resulted in a peak output power of $879-\mathrm{W}$ (with constant drive), a $>25 \%$ increase of $6430 \mathrm{~N} 1$ when compared with NRL-3. VTA-6430N2 produced a maximum output power of $963-\mathrm{W}$ before DIO was observed.

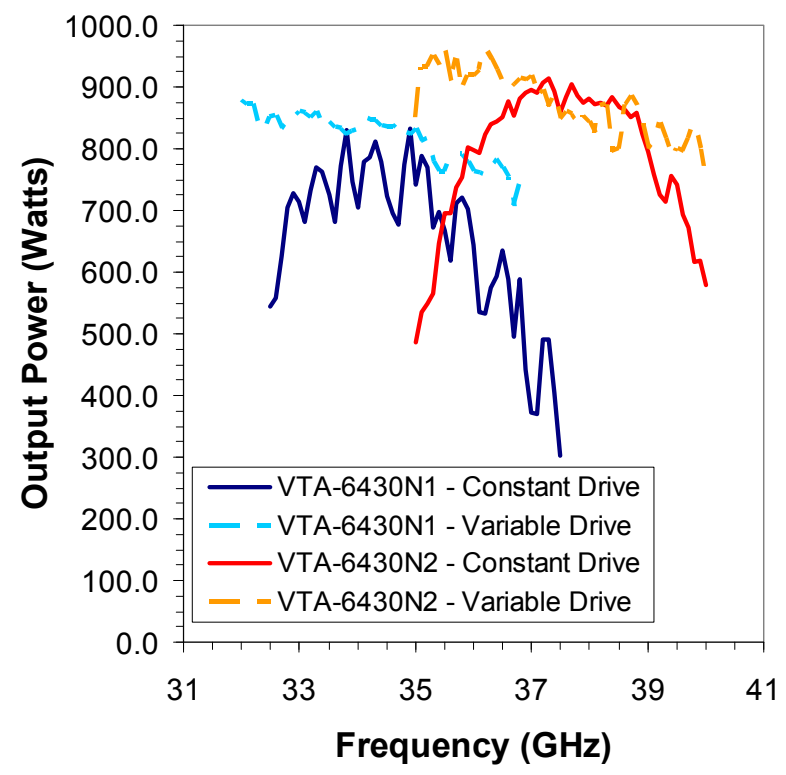

Figure 2: VTA-6430N1, 6430N2 output power vs. frequency for constant input drive (solid line) and variable input drive (dashed line)

\section{Summary}

We have designed, manufactured and successfully tested a series of Ka-band CCTWT prototype amplifiers that have extended the state-of-the-art in power, bandwidth, and stability performance. To date, both the VTA-6430N1 and VTA-6430N2 have demonstrated over 700-W peak power (879-W and 963-W maximum power) over a $5-\mathrm{GHz}$ bandwidth $\left(f_{0}=35 \mathrm{GHz}\right.$ and $\left.37.5 \mathrm{GHz}\right)$ respectively.

\section{Acknowledgements}

This work was supported by U.S. Office of Naval Research.

\section{References}

1. Chernin, D. et al., "Large signal multi-frequency simulation of coupled-cavity TWTs," IEEE Transactions On Electron Devices, vol. 58, no. 4, pp 1229-1240, April 2001.

2. Vlasov, A.N. et al., "2D modeling of beam-wave interaction in coupled cavity TWT," 2010 IEEE Int'l Vacuum Electronics Conf., Monterey, CA, May 1820, 2010, pp. 405-406.

3. Petillo, J.J. et al., "Recent developments to the MICHELLE 2-D/3-D electron gun and collector modeling code," IEEE Trans. Electron Devices, vol. 52(5), pp. 742-748, May 2005. 